\title{
Remarks on the Solution of Laplace's Differential Equation and Fractional Differential Equation of That Type
}

\author{
Tohru Morita $^{1}$, Ken-ichi Sato ${ }^{2}$ \\ ${ }^{1}$ Tohoku University, Sendai, Japan \\ ${ }^{2}$ College of Engineering, Nihon University, Koriyama, Japan \\ Email: senmm@jcom.home.ne.jp
}

Received June 24, 2013; revised July 24, 2013; accepted July 31, 2013

Copyright (C) 2013 Tohru Morita, Ken-ichi Sato. This is an open access article distributed under the Creative Commons Attribution License, which permits unrestricted use, distribution, and reproduction in any medium, provided the original work is properly cited.

\begin{abstract}
We discuss the solution of Laplace's differential equation by using operational calculus in the framework of distribution theory. We here study the solution of that differential Equation with an inhomogeneous term, and also a fractional differential equation of the type of Laplace's differential equation.
\end{abstract}

Keywords: Laplace's Differential Equation; Kummer's Differential Equation; Fractional Differential Equation; Inhomogeneous Equation; Distribution Theory; Operational Calculus

\section{Introduction}

Yosida $[1,2]$ discussed the solution of Laplace's differential equation (DE), which is a linear DE with coefficients which are linear functions of the variable. The DE which he takes up is

$$
\begin{aligned}
& \left(a_{2} t+b_{2}\right) y^{\prime \prime}(t)+\left(a_{1} t+b_{1}\right) y^{\prime}(t) \\
& +\left(a_{0} t+b_{0}\right) y(t)=0, \quad t>0,
\end{aligned}
$$

where $a_{l}$ and $b_{l}$ for $l=0,1,2$ are constants. His discussion is based on Mikusiński's operational calculus [3].

In our preceding papers $[4,5]$, we discuss the initial-value problem of linear fractional differential equation (fDE) with constant coefficients, in terms of distribution theory. The formulation is given in the style of primitive operational calculus, solving a Volterra integral equation with the aid of Neumann series.

Yosida [1,2] studied the homogeneous Equation (1.1), where he gave only one of the solutions by that method. One of the purposes of the present paper is to give the recipe of obtaining the solution of the inhomogeneous equation as well as the homogeneous one, in the style of operational calculus in the framework of distribution theory. With the aid of that recipe, we show how the set of two solutions of the homogeneous equation is attained.

Another purpose of this paper is to discuss the solution of an fDE of the type of Laplace's DE, which is a linear fDE with coefficients which are linear functions of the variable. In place of (1.1), we consider

$$
\begin{aligned}
& \left(a_{2} t+b_{2}\right) \cdot{ }_{0} D_{R}^{2 \sigma} u(t)+\left(a_{1} t+b_{1}\right) \cdot{ }_{0} D_{R}^{\sigma} u(t) \\
& +\left(a_{0} t+b_{0}\right) u(t)=f(t), t>0,
\end{aligned}
$$

for $\sigma=1$ and $\sigma=1 / 2$. Here ${ }_{0} D_{R}^{\sigma} u(t)$ for $\sigma \in \mathbb{R}_{>0}$ is the Riemann-Liouville (R-L) fractional derivative defined in Section 2. We use $\mathbb{R}$ to denote the set of all real numbers, and $\mathbb{R}_{>b}:=\{x \in \mathbb{R} \mid x>b\}$. When $\sigma$ is equal to an integer $n \in \mathbb{Z}_{>0},{ }_{0} D_{R}^{n} u(t)=\frac{\mathrm{d}^{n}}{\mathrm{~d} t^{n}} u(t)$. When $\sigma=1,(1.2)$ is the inhomogeneous DE for (1.1). We use $\mathbb{Z}$ to denote the set of all integers, and $\mathbb{Z}_{>a}:=\{n \in \mathbb{Z} \mid n>a\}$ and $\mathbb{Z}_{[a, b]}:=\{n \in \mathbb{Z} \mid a \leq n \leq b\}$ for $a, b \in \mathbb{Z}$ satisfying $a<b$. We use $\lceil x\rceil$ for $x \in \mathbb{R}$, to denote the least integer that is not less than $x$.

In Section 2, we prepare the definition of R-L fractional derivative and then explain how (1.2) is converted into a $\mathrm{DE}$ or an $\mathrm{fDE}$ of a distribution in distribution theory. A compact definition of distributions in the space $\mathcal{D}_{R}^{\prime}$ and their fractional integral and derivative are described in Appendix A. A proof of a lemma in Section 2 is given in Appendix B. After these preparation, a recipe is given to be used in solving a DE with the aid of operational culculus in Section 3. In this recipe, the solution is 
obtained only when $a_{2} \neq 0$ and $b_{2}=0$. When $\sigma=\frac{1}{2}$, $b_{1}=0$ is also required. An explanation of this fact is given in Appendices $\mathbf{C}$ and $\mathbf{D}$. In Section 4, we apply the recipe to the DE where $a_{0}=0$, of which special one is Kummer's DE. This is an example which Yosida $[1,2]$ takes up. In Section 5, we apply the recipe to the fDE with $\sigma=\frac{1}{2}$, assuming $a_{0}=0$.

The discussion is done in the style of our preceding papers $[4,5]$.

\section{Formulas}

We use Heaviside's step function, which we denote by $H(t)$. When $f(t)$ is defined on $\mathbb{R}_{>b}, f(t) H(t-b)$ is assumed to be equal to $f(t)$ when $t>b$ and to 0 when $t \leq b$.

\subsection{Riemann-Liouville Fractional Integral and Derivative}

Let $u(t) H(t-b)$ be locally integrable on $\mathbb{R}$. We then define the R-L fractional integral ${ }_{b} D_{R}^{-v} u(t)$ of order $v \in \mathbb{R}_{>0}$ by

$$
{ }_{b} D_{R}^{-v} u(t)=\frac{1}{\Gamma(v)} \int_{b}^{t}(t-x)^{v-1} u(x) \mathrm{d} x, t>b,
$$

where $\Gamma(v)$ is the gamma function. The thus-defined ${ }_{b} D_{R}^{-v} u(t)$ is locally integrable on $\mathbb{R}_{>b}$, and ${ }_{b} D_{R}^{-v} u(b+)=0$ if $v \geqq 1$.

We define the R-L fractional derivative ${ }_{b} D_{R}^{\beta} u(t)$ of order $\beta \in \mathbb{R}_{>0}$, by

$$
{ }_{b} D_{R}^{\beta} u(t)=\frac{\mathrm{d}^{N}}{\mathrm{~d} t^{N}}\left[{ }_{b} D_{R}^{\beta-N} u(t)\right], t>b,
$$

if it exists, where $N=\lceil\beta\rceil$, and ${ }_{b} D_{R}^{0} u(t)=u(t)$ for $t>b$. fied.

We now assume that the following condition is satis-

Condition A $u(t) H(t)$ is locally integrable on $\mathbb{R}$, and there exists ${ }_{0} D_{R}^{\beta} u(t)$ for $t \geqq 0$, and ${ }_{0} D_{R}^{n+\beta-N} u(t)$ for $n \in \mathbb{Z}_{[0, N-1]}$ are continuous and differentiable at $t>0$, where $N=\lceil\beta\rceil$. We then assume that there exists a finite value

$$
u_{\beta-k-1}:={ }_{0} D_{R}^{\beta-k-1} u(0+),
$$

for every $k \in \mathbb{Z}_{[0, N-1]}$.

Because of this condition, the Taylor series expansion of ${ }_{0} D_{R}^{\beta-N} u(t)$ is given by

$$
{ }_{0} D_{R}^{\beta-N} u(t)=\sum_{n=0}^{N-1} u_{\beta-N+n} \cdot \frac{t^{n}}{n !}+w(t), t>0,
$$

where $w(t)$ is a function of $o\left(t^{N-1}\right)$ as $t \rightarrow 0+$, so that $w(t) / t^{N-1} \rightarrow 0$ as $t \rightarrow 0+$. By comparing (2.2) and (2.4), we obtain ${ }_{0} D_{R}^{\beta} u(t)=\frac{\mathrm{d}^{N}}{\mathrm{~d} t^{N}} w(t)$.

\subsection{Fractional Integral and Derivative of a Distribution}

We consider distributions belonging to $\mathcal{D}^{\prime}$. When a function $h(t)$ is locally integrable on $\mathbb{R}$ and has a support bounded on the left, it belongs to $\mathcal{D}^{\prime}{ }_{R}$ and is called a regular distribution. The distributions in $\mathcal{D}^{\prime}{ }_{R}$ are called right-sided distributions.

A compact formal definition of a distribution in $\mathcal{D}^{\prime}{ }_{R}$ and its fractional integral and derivative is given in Appendix $\mathbf{A}$.

Let $f(t) H(t)$ be a regular distribution. Then $\left[{ }_{0} D_{R}^{-v} f(t)\right] H(t)$ for $v \geq 0$ is also a regular distribution, and distribution $D^{-v}[f(t) H(t)]$ is defined by

$$
D^{-v}[f(t) H(t)]=\left[{ }_{0} D_{R}^{-v} f(t)\right] H(t), \quad v \geq 0 .
$$

Let $N \in \mathbb{Z}_{>0}$, and let $h(t)$ be such a regular distribution that $\frac{\mathrm{d}^{n}}{\mathrm{~d} t^{n}} h(t)$ is continuous and differentiable on $\mathbb{R}$, for every $n \in \mathbb{R}_{[0, N-1]}$. Then $D^{N} h(t)$ is defined by

$$
D^{N} h(t)=\frac{\mathrm{d}^{N}}{\mathrm{~d} t^{N}} h(t) .
$$

$$
\text { Let } \frac{\mathrm{d}^{n}}{\mathrm{~d} t^{n}}\left[\left\{{ }_{0} D_{R}^{\beta-N} f(t)\right\} H(t)\right], \text { for } \beta \in \mathbb{R}_{>0} \quad \text { and }
$$

$N=\lceil\beta\rceil$, be continuous and differentiable on $\mathbb{R}$, for every $n \in \mathbb{R}_{[0, N-1]}$. Then

$$
D^{\beta}[f(t) H(t)]=\left[{ }_{0} D_{R}^{\beta} f(t)\right] H(t) .
$$

When $h(t)$ is a regular distribution, $D^{v} h(t)$ is defined for all $v \in \mathbb{R}$.

Lemma 1 For $h(t) \in \mathcal{D}^{\prime}{ }_{R}$, the index law:

$$
D^{v} D^{\beta} h(t)=D^{v+\beta} h(t),
$$

is valid for every $v, \beta \in \mathbb{R}$.

Dirac's delta function $\delta(t)$ is the distribution defined by $\delta(t)=D H(t)$.

Lemma 2 Let $g_{v}(t)=\frac{1}{\Gamma(v)} t^{v-1} H(t)$ for $v \in \mathbb{R}_{>0}$.

Then

$$
g_{v}(t)=D^{-v} \delta(t), \quad v>0 .
$$

Proof By putting $b=0, v \in \mathbb{R}_{>0}$, and $u(t)=1$ in (2.1), we obtain ${ }_{0} D_{R}^{-v} 1=\frac{1}{\Gamma(v+1)} t^{v}$. By (2.5), we then have $D^{-v} H(t)=\frac{1}{\Gamma(v+1)} t^{v} H(t)$. By applying $D$ to 
this and using (2.6) and (2.8), we obtain (2.9).

We now adopt the following condition.

Condition B $u(t) H(t)$ and $f(t) H(t)$ are expressed as a linear combination of $g_{v}(t)$ for $v>0$.

Then $u(t) H(t)$ and $f(t) H(t)$ are expressed as

$$
\begin{aligned}
& u(t) H(t)=\hat{u}(D) \delta(t), \\
& f(t) H(t)=\hat{f}(D) \delta(t) .
\end{aligned}
$$

Lemma 3 Let ${ }_{0} D_{R}^{\beta} u(t)$ exist for $\beta \in \mathbb{R}_{>0}$. Then the products $u(t) H(t)$ and $\left[{ }_{0} D_{R}^{\beta} u(t)\right] H(t)$ belong to $\mathcal{D}_{R}^{\prime}$, and they are related by

$$
\begin{aligned}
D^{\beta}[u(t) H(t)] & =\left[{ }_{0} D_{R}^{\beta} u(t)\right] H(t) \\
& +\sum_{k=0}^{\lceil\beta\rceil-1} u_{\beta-k-1} \cdot D^{k} \delta(t) .
\end{aligned}
$$

Proof We obtain (2.11) from (2.4) by multiplying $H(t)$ from the right and then applying $D^{N}$. We first note $D^{\beta-N}[u(t) H(t)]=\left[{ }_{0} D_{R}^{\beta-N} u(t)\right] H(t)$ due to (2.5). Applying $D^{N}$ to this, we obtain the lefthand side of (2.11), and hence from the lefthand side of (2.4). We next note that

$$
\begin{aligned}
D^{N}[w(t) H(t)] & =\frac{\mathrm{d}^{N}}{\mathrm{~d} t^{N}}[w(t) H(t)] \\
& =\left[\frac{\mathrm{d}^{N}}{\mathrm{~d} t^{N}} w(t)\right] H(t)
\end{aligned}
$$

due to (2.6) and ${ }_{0} D_{R}^{\beta} u(t)=\frac{\mathrm{d}^{N}}{\mathrm{~d} t^{N}} w(t)$ as noted after (2.4). Thus we obtain the first term on the righthand side of (2.11) from the last term of (2.4). As to the remaining terms, we only use (2.9).

Lemma 4 Let $\beta \in \mathbb{R}$. Then

$$
t \cdot D^{\beta} \delta(t)=-\beta \cdot D^{\beta-1} \delta(t)=-\frac{\partial}{\partial D} D^{\beta} \delta(t) .
$$

The last derivative with respect to $D$ is taken regarding $D$ as a variable.

Proof of Lemma 4 for $\beta \in \mathbb{R}_{<0}$. Let $\beta=-v$, $v \in \mathbb{R}_{>0}$. Then by (2.9), we have

$$
\begin{aligned}
t \cdot D^{-v} \delta(t) & =t \cdot g_{v}(t)=\frac{v}{\Gamma(v+1)} t^{v} H(t) \\
& =v D^{-v-1} \delta(t)=-\frac{\partial}{\partial D} D^{-v} \delta(t),
\end{aligned}
$$

by using (2.9) repeatedly.

A proof of this lemma for $\beta \in \mathbb{R}_{>0}$ is given in Appendix $B$.

The following lemma is a consequence of this lemma.

Lemma 5 Let $u(t) H(t)$ satisfy Condition B. Then

$$
t \cdot u(t) H(t)=t \cdot \hat{u}(D) \delta(t)=-\frac{\partial}{\partial D} \hat{u}(D) \delta(t) .
$$

\section{Lemma 6}

$$
\begin{aligned}
D^{\lambda}[t \cdot u(t) H(t)] & =t \cdot D^{\lambda}[u(t) H(t)] \\
& +\lambda D^{\lambda-1}[u(t) H(t)] .
\end{aligned}
$$

Proof By using (2.10) and (2.13), we obtain

$$
\begin{aligned}
& t \cdot D^{\lambda}[u(t) H(t)]=-\frac{\partial}{\partial D} D^{\lambda}[\hat{u}(D) \delta(t)] \\
& =-\lambda D^{\lambda-1}[\hat{u}(D) \delta(t)]-D^{\lambda}\left[\frac{\partial}{\partial D} \hat{u}(D) \delta(t)\right] \\
& =-\lambda D^{\lambda-1}[u(t) H(t)]+D^{\lambda}[t \cdot u(t) H(t)] .
\end{aligned}
$$

\section{Recipe of Solving Laplace's DE and fDE of That Type}

We now express the DE/fDE (1.2) to be solved, as follows:

$$
\sum_{l=0}^{m}\left(a_{l} t+b_{l}\right) \cdot{ }_{0} D_{R}^{l \sigma} u(t)=f(t), \quad t>0,
$$

where $\sigma=\frac{1}{2}$ or $\sigma=1$, and $m=2$. In Sections 4 and 5, we study this DE for $\sigma=1$ and this fDE for $\sigma=\frac{1}{2}$, respectively.

\subsection{Deform to DE/fDE for Distribution}

Using Lemma 3, we express (3.1) as

$$
\begin{aligned}
& \sum_{l=0}^{m}\left(a_{l} t+b_{l}\right) \cdot D^{l \sigma}[u(t) H(t)] \\
& =f(t) H(t)+v(t) H(t),
\end{aligned}
$$

where

$$
v(t) H(t):=\sum_{l=1}^{m}\left[\left(a_{l} t+b_{l}\right) \sum_{k=0}^{\lceil l \sigma\rceil-1} u_{l \sigma-k-1} \cdot D^{k} \delta(t)\right] .
$$

\subsection{Solution via Operational Calculus}

By using (2.10) and (2.13), we express (3.2) as

$$
\begin{aligned}
& -\sum_{l=0}^{m} a_{l} \cdot \frac{\partial}{\partial D}\left[D^{l \sigma} \hat{u}(D) \delta(t)\right]+\sum_{l=0}^{m} b_{l} \cdot D^{l \sigma} \hat{u}(D) \delta(t) \\
& =-A(D)\left[\frac{\partial}{\partial D} \hat{u}(D) \delta(t)\right]+B(D)[\hat{u}(D) \delta(t)] \\
& =\hat{f}(D) \delta(t)+\hat{v}(D) \delta(t),
\end{aligned}
$$

where

$$
\begin{aligned}
& A(D)=\sum_{l=0}^{m} a_{l} \cdot D^{l \sigma}, \\
& B(D)=\sum_{l=0}^{m}\left(-l \cdot \sigma \cdot a_{l} \cdot D^{l \sigma-1}+b_{l} \cdot D^{l \sigma}\right),
\end{aligned}
$$




$$
\begin{aligned}
& \hat{v}(D)= \\
& \sum_{l=1}^{m}\left[-a_{l} \sum_{k=0}^{\lceil l \sigma\rceil-1} k \cdot u_{l \sigma-k-1} \cdot D^{k-1}+b_{l} \sum_{k=0}^{\lceil l \sigma\rceil-1} u_{l \sigma-k-1} \cdot D^{k}\right] .
\end{aligned}
$$

In order to solve the Equation (3.4) for $\hat{u}(D) \delta(t)$, we solve the following equation for function $\hat{u}(x)$ of real variable $x$ :

$$
-A(x) \frac{\mathrm{d}}{\mathrm{d} x} \hat{u}(x)+B(x) \hat{u}(x)=\hat{f}(x)+\hat{v}(x) .
$$

Lemma 7 The complementary solution (C-solution) of Equation (3.7) is given by $\hat{u}(x)=C_{1} \cdot \hat{\phi}(x)$, where $C_{1}$ is an arbitrary constant and

$$
\hat{\phi}(x)=C_{2} \cdot \exp \left(\int^{x} \frac{B(\xi)}{A(\xi)} \mathrm{d} \xi\right),
$$

where the integral is the indefinite integral and $C_{2}$ is any constant.

Lemma 8 Let $\hat{\phi}(x)$ be the C-solution of (3.7), and let the particular solution (P-solution) of (3.7) be $\hat{u}_{v}^{*}(x)$ when the inhomogeneous part is $x^{-v}$ for $v \in \mathbb{R}$. Then

$$
\hat{u}_{v}^{*}(x)=-\hat{\phi}(x) \int^{x} \frac{\xi^{-v}}{A(\xi) \hat{\phi}(\xi)} \mathrm{d} \xi+C_{3} \cdot \hat{\phi}(x),
$$

where $C_{3}$ is any constant.

Since $f(t) H(t)$ satisfies Condition B and $\hat{v}(D)$ is given by (3.6), the P-solution $\hat{u}(x)$ of (3.7) is expressed as a linear combination of $\hat{u}_{v}^{*}(x)$ for $v \in \mathbb{R}_{>0}$ and $\hat{u}_{-k}^{*}(x)$ for $k \in \mathbb{Z}_{>-1}$.

From the solution $\hat{u}(x)$ of (3.7), $\hat{u}(D)$ is obtained by substituting $x$ by $D$. Then we confirm that (3.4) is satisfied by that $\hat{u}(D)$ applied to $\delta(t)$.

\subsection{Neumann Series Expansion}

Finally the obtained expression of $\hat{u}(D)$ is expanded into the sum of terms of negative powers of $D$, and then we obtain the solution $\hat{u}(D) \delta(t)$ of (3.4). If the obtained $\hat{u}(D)$ is a linear combination of $D^{-v}$ for $v \in \mathbb{R}_{>0}, \hat{u}(D) \delta(t)$ is converted to the solution $u(t) H(t)$ of (3.2) by using (2.10) and (2.9). It becomes a solution $u(t)$ of (3.1) for $t>0$.

\subsection{Recipe of Obtaining the Solution of (3.1)}

1) We prepare the data: $\hat{f}(D)$ by (2.10), and $A(x)$, $B(x)$ and $\hat{v}(D)$ by (3.5) and (3.6).

2) We obtain $\hat{\phi}(x)$ by (3.8). If $\hat{v}(D)=0$, the Csolution of (3.1) is given by

$$
u(t) H(t)=C_{1} \cdot \hat{\phi}(D) \delta(t), t>0 .
$$

3) If $\hat{f}(D) \neq 0$ or $\hat{v}(D) \neq 0$, we obtain $\hat{u}_{v}^{*}(x)$ given by (3.9).
4) If $\hat{v}(D)=\sum_{k=0}^{\lceil m \sigma\rceil-1} c_{k} D^{k} \neq 0$, the C-solution of (3.1) is given by

$$
u(t) H(t)=C_{1} \cdot \hat{\phi}(D) \delta(t)+\sum_{k=0}^{\lceil m \sigma\rceil-1} c_{k} \hat{u}_{-k}^{*}(D) \delta(t), t>0,
$$

where $c_{k}$ are constants.

5) If $\hat{f}(D)=\sum_{k=1}^{\infty} d_{k} D^{-v_{k}} \neq 0$, the P-solution of (3.1) is given by

$$
u(t) H(t)=\sum_{k=1}^{\infty} d_{k} \hat{u}_{v_{k}}^{*}(D) \delta(t), t>0,
$$

where $v_{k} \in \mathbb{R}_{>0}$ and $d_{k}$ are constants.

\subsection{Solution of (3.1) from the Solution of (3.7)}

In the above recipe, we first obtain the C-solution of (3.7), that is $\hat{u}(x)=C_{1} \cdot \hat{\phi}(x)$. It gives the C-solution $\hat{u}(D) \delta(t)$ of (3.4) and hence the C-solutions $u(t) H(t)$ of (3.2) and $u(t)$ of (3.1).

We next obtain the P-solution $\hat{u}_{v}^{*}(x)$ of (3.7) when the inhomogeneous part is $x^{-v}$ for $v \in \mathbb{R}$. As noted above, the P-solutions $\hat{u}(x)$ of (3.7) for $\hat{f}(x)$ and for $\hat{v}(x)$, are expressed as a linear combination of $\hat{u}_{v}^{*}(x)$ for $v \in \mathbb{R}_{>0}$ and of $\hat{u}_{-k}^{*}(x)$ for $k \in \mathbb{Z}_{>-1}$, respectively. The sum of the P-solutions $\hat{u}(x)$ of (3.7) for $\hat{f}(x)$ and for $\hat{v}(x)$ gives the P-solution $\hat{u}(D) \delta(t)$ of (3.4) and hence the P-solution $u(t) H(t)$ of (3.2). The C-solution $u(t)$ of (3.1) comes from the C-solution of (3.7) and the P-solution of (3.7) for $\hat{v}(x)$.

\subsection{Remarks}

When we obtain $\hat{u}(D)$ at the end of Section 3.2, we must examine whether it is compatible with Condition B. We will find that if $b_{l} \neq 0$ for $l \sigma>m \sigma-1$, the obtained $\hat{u}(D)$ is not acceptable. Hence we have to solve the problem, assuming that $b_{l}=0$ for all $l>m-1 / \sigma$. When $\sigma=\frac{1}{2}$ and $m=2$, we put $b_{2}=b_{1}=0$. When $\sigma=1$ and $m=2$, we put $b_{2}=0$. Discussion of this problem is given in Appendices $\mathbf{C}$ and $\mathbf{D}$.

\section{Laplace's and Kummer's DE}

We now consider the case of $\sigma=1, m=2, a_{2} \neq 0$, $a_{1} \neq 0, a_{0}=0$ and $b_{2}=0$. Then (3.1) reduces to

$$
\begin{aligned}
& a_{2} t \cdot \frac{\mathrm{d}^{2}}{\mathrm{~d} t^{2}} u(t)+\left(a_{1} t+b_{1}\right) \cdot \frac{\mathrm{d}}{\mathrm{d} t} u(t)+b_{0} \cdot u(t) \\
& =f(t), \quad t>0 .
\end{aligned}
$$

By (3.5) and (3.6), $A(x), B(x)$ and $\hat{v}(x)$ are

$$
\begin{aligned}
& A(x)=a_{2} x^{2}+a_{1} x=a_{2} x(x+\alpha), \\
& B(x)=\left(b_{1}-2 a_{2}\right) x+b_{0}-a_{1},
\end{aligned}
$$




$$
\hat{v}(x)=-a_{2} u_{0}+b_{1} u_{0},
$$

where $\alpha=a_{1} / a_{2}$.

\subsection{Complementary Solution of (3.7), (3.4) and (3.2)}

In order to obtain the C-solution $\hat{\phi}(x)$ of (3.7) by using (3.8), we express $B(x) / A(x)$ as follows:

$$
\frac{B(x)}{A(x)}=\frac{\gamma_{1}}{x}+\frac{\gamma_{2}}{x+\alpha}
$$

where

$$
\gamma_{1}+\gamma_{2}=\frac{b_{1}}{a_{2}}-2, \quad \gamma_{1}=\frac{b_{0}}{a_{1}}-1, \quad \gamma_{2}=\frac{b_{1}}{a_{2}}-\frac{b_{0}}{a_{1}}-1 .
$$

$B(x)$ is now expressed as

$$
B(x)=a_{2}\left(\gamma_{1}+\gamma_{2}\right) x+a_{1} \gamma_{1} .
$$

By using (3.8), we obtain

$$
\begin{aligned}
\hat{\phi}(x) & =x^{\gamma_{1}}(x+\alpha)^{\gamma_{2}}=x^{\gamma_{1}+\gamma_{2}}\left(1+\alpha x^{-1}\right)^{\gamma_{2}} \\
& =\sum_{n=0}^{\infty}\left(\begin{array}{c}
\gamma_{2} \\
n
\end{array}\right) \alpha^{n} x^{-n+\gamma_{1}+\gamma_{2}}
\end{aligned}
$$

where $\left(\begin{array}{l}\gamma \\ n\end{array}\right)=\frac{(-1)^{n}(-\gamma)_{n}}{n !}$ for $\gamma \in \mathbb{R}$ and $n \in \mathbb{Z}_{>-1}$ are the binomial coefficients. Here $(a)_{n}=\prod_{k=0}^{n-1}(a+k)$ for $a \in \mathbb{R}$ and $n \in \mathbb{Z}_{>0}$, and $(a)_{0}=1$.

The C-solution of (3.4) is given by

$$
\begin{aligned}
\hat{u}(D) \delta(t) & =C_{1} \cdot \hat{\phi}(D) \delta(t) \\
& =C_{1} \cdot D^{\gamma_{1}+\gamma_{2}}\left(1+\alpha D^{-1}\right)^{\gamma_{2}} \delta(t) \\
& =C_{1} \sum_{n=0}^{\infty}\left(\begin{array}{c}
\gamma_{2} \\
n
\end{array}\right) \alpha^{n} D^{-n+\gamma_{1}+\gamma_{2}} \delta(t) .
\end{aligned}
$$

If $\gamma_{1}+\gamma_{2}<0$, Condition B is satisfied. Then by using (2.9), we obtain the C-solution of (3.2):

$$
\begin{aligned}
u(t) H(t) & =C_{1} \cdot \phi(t)=C_{1} \sum_{n=0}^{\infty}\left(\begin{array}{c}
\gamma_{2} \\
n
\end{array}\right) \alpha^{n} \frac{1}{\Gamma\left(n-\gamma_{1}-\gamma_{2}\right)} t^{n-\gamma_{1}-\gamma_{2}-1} H(t) \\
& =C_{1} \frac{1}{\Gamma\left(-\gamma_{1}-\gamma_{2}\right)} \sum_{n=0}^{\infty} \frac{\left(-\gamma_{2}\right)_{n}}{n !\left(-\gamma_{1}-\gamma_{2}\right)_{n}}(-\alpha t)^{n} \cdot t^{-\gamma_{1}-\gamma_{2}-1} H(t) \\
& =C_{1} \frac{1}{\Gamma\left(-\gamma_{1}-\gamma_{2}\right)} t^{-\gamma_{1}-\gamma_{2}-1} \cdot{ }_{1} F_{1}\left(-\gamma_{2} ;-\gamma_{1}-\gamma_{2} ;-\alpha t\right) H(t)
\end{aligned}
$$

Remark 1 In [6,7], Kummer's DE is given, which is equal to the DE (4.1) for $a_{2}=1, a_{1}=-1, b_{1}=c$ and $b_{0}=-a$. In this case,

$$
\begin{aligned}
& \gamma_{2}=c-a-1, \quad \gamma_{1}+\gamma_{2}=c-2, \\
& \gamma_{1}=a-1, \quad \alpha=-1 .
\end{aligned}
$$

We then confirm that the expression (4.8) agrees with one of the C-solutions of Kummer's DE given in those books.

\subsection{Particular Solution of (3.7)}

We now obtain the P-solution of (3.7) when the inhomogeneous part is equal to $x^{-v}$ for $v \geq 0$.

When the C-solution of (3.7) is $\hat{\phi}(x)$ given by (4.6), the P-solution of (3.7) is given by (3.9). By using (4.2) and (4.6), we obtain

$$
\begin{aligned}
\hat{u}_{v}^{*}(x) & =-x^{\gamma_{1}}(x+\alpha)^{\gamma_{2}} \int^{x} \frac{\xi^{-v}}{a_{2} \xi^{1+\gamma_{1}}(\xi+\alpha)^{1+\gamma_{2}}} \mathrm{~d} \xi+C_{3} \cdot \hat{\phi}(x) \\
& =-\frac{1}{a_{2}} x^{\gamma_{1}+\gamma_{2}}\left(1+\alpha x^{-1}\right)^{\gamma_{2}} \int^{x} \xi^{-2-\gamma_{1}-\gamma_{2}-v}\left(1+\alpha \xi^{-1}\right)^{-1-\gamma_{2}} \mathrm{~d} \xi+C_{3} \cdot \hat{\phi}(x) \\
& =-\frac{1}{a_{2}} \sum_{n=0}^{\infty}\left(\begin{array}{c}
\gamma_{2} \\
n
\end{array}\right) \alpha^{n} x^{-n+\gamma_{1}+\gamma_{2}} \int^{x} \sum_{m=0}^{\infty}\left(\begin{array}{c}
-1-\gamma_{2} \\
m
\end{array}\right) \alpha^{m} \xi^{-m-\gamma_{1}-\gamma_{2}-2-v} \mathrm{~d} \xi+C_{3} \cdot \hat{\phi}(x) \\
& =\frac{1}{a_{2}} \sum_{n=0}^{\infty}\left(\begin{array}{c}
\gamma_{2} \\
n
\end{array}\right) \alpha^{n} x^{-n} \sum_{m=0}^{\infty}\left(\begin{array}{c}
-1-\gamma_{2} \\
m
\end{array}\right) \alpha^{m} \frac{1}{m+\gamma_{1}+\gamma_{2}+1+v} x^{-m-1-v} \\
& =\frac{1}{a_{2}} \sum_{n=0}^{\infty}{ }_{n} C_{\gamma_{2}, \gamma_{1}+1+v}^{*} \cdot \alpha^{n} x^{-n-1-v},
\end{aligned}
$$


where

$$
{ }_{n} C_{p_{1}, p_{2}}^{*}=\sum_{k=0}^{n}\left(\begin{array}{c}
p_{1} \\
k
\end{array}\right)\left(\begin{array}{c}
-1-p_{1} \\
n-k
\end{array}\right) \frac{1}{n-k+p_{1}+p_{2}} .
$$

Lemma $9{ }_{n} C_{p_{1}, p_{2}}^{*}$ defined by (4.11) is expressed as

$$
{ }_{n} C_{p_{1}, p_{2}}^{*}=(-1)^{n} \frac{1}{p_{1}+p_{2}} \cdot \frac{\left(p_{2}\right)_{n}}{\left(1+p_{1}+p_{2}\right)_{n}} \text {. }
$$

Proof Equation (4.10) shows that the P-solution $\hat{u}_{v}^{*}(x)$ of (3.7) is now expressed as

$$
\hat{u}_{v}^{*}(x)=\frac{1}{a_{2}} \sum_{n=0}^{\infty} c_{n} \alpha^{n} x^{-n-1-v},
$$

where $c_{n}={ }_{n} C_{\gamma_{2}, \gamma_{1}+v+1}^{*}$. Substituting this into (3.7), we obtain an equation which states that a power series of $x^{-1}$ is equal to 0 . By the condition that the coefficient of every power must be 0 , we obtain a recurrence equation for the coefficients $c_{n}$ :

$$
\begin{aligned}
& c_{0}=\frac{1}{1+v+\gamma_{1}+\gamma_{2}}, \\
& c_{n}=-\frac{v+n+\gamma_{1}}{1+v+n+\gamma_{1}+\gamma_{2}} c_{n-1}, \quad n \in \mathbb{Z}_{>0} .
\end{aligned}
$$

By using this repeatedly, we have

$$
c_{n}=(-1)^{n} \frac{\left(v+1+\gamma_{1}\right)_{n}}{\left(2+v+\gamma_{1}+\gamma_{2}\right)_{n}} c_{0}, \quad n \in \mathbb{Z}_{>0} .
$$

By comparing (4.10), (4.13) and (4.16), we obtain (4.12).

\subsection{Particular Solution of (3.2)}

Equation (4.10) shows that if the inhomogeneous part is $D^{-v} \delta(t)$ for $v \geq 0$, the P-solution of (3.2) is given by

$$
\begin{aligned}
& u_{v}^{*}(t) H(t) \\
& =\frac{1}{a_{2}} \sum_{n=0}^{\infty}{ }_{n} C_{\gamma_{2}, \gamma_{1}+1+v}^{*} \frac{1}{\Gamma(n+1+v)} \alpha^{n} \cdot t^{n+v} H(t) .
\end{aligned}
$$

By using (4.12) in (4.17), we obtain

$$
\begin{aligned}
& u_{v}^{*}(t) H(t) \\
= & \frac{1}{a_{2}\left(1+v+\gamma_{1}+\gamma_{2}\right) \Gamma(v+1)} t^{v} \\
& \cdot \sum_{n=0}^{\infty} \frac{\left(v+1+\gamma_{1}\right)_{n}(1)_{n}}{\left(2+v+\gamma_{1}+\gamma_{2}\right)_{n}(v+1)_{n} n !}(-\alpha t)^{n} H(t) \\
= & \frac{1}{a_{2}\left(1+v+\gamma_{1}+\gamma_{2}\right) \Gamma(v+1)} t^{v} \\
{ }_{2} & F_{2}\left(v+1+\gamma_{1}, 1 ; 2+v+\gamma_{1}+\gamma_{2}, v+1 ;-\alpha t\right) H(t) .
\end{aligned}
$$

\subsection{Complementary Solution of (4.1)}

By (4.3), $\hat{v}(x)=-\left(a_{2}-b_{1}\right) u_{0}=a_{2}\left(\gamma_{1}+\gamma_{2}+1\right) u_{0}$. When the inhomogeneous part is $\hat{v}(x) \neq 0$, the $\mathrm{P}$-solution of (3.7) is given by

$$
\hat{u}(x)=a_{2} \cdot\left(\gamma_{1}+\gamma_{2}+1\right) \cdot u_{0} \cdot \hat{u}_{0}^{*}(x) .
$$

By using (4.18) for $v=0$, we obtain

$$
\begin{aligned}
u(t) H(t) & =a_{2} \cdot\left(\gamma_{1}+\gamma_{2}+1\right) \cdot u_{0} \cdot u_{0}^{*}(t) H(t) \\
& =u_{0} \sum_{n=0}^{\infty} \frac{\left(1+\gamma_{1}\right)_{n}}{\left(2+\gamma_{1}+\gamma_{2}\right)_{n} n !}(-\alpha t)^{n} H(t) \\
& =u_{0} \cdot{ }_{1} F_{1}\left(1+\gamma_{1} ; 2+\gamma_{1}+\gamma_{2} ;-\alpha t\right) H(t) .
\end{aligned}
$$

Proposition 1 Let $u_{0} \neq 0$ and $\gamma_{1}+\gamma_{2}=\frac{b_{1}}{a_{2}}-2<-1$. Then the complementary solution of (4.1), multiplied by $H(t)$, is given by the sum of the righthand sides of (4.8) and of (4.21).

Remark 2 As stated in Remark 1, in [6,7], the result for $a_{2}=1, a_{1}=-1, b_{1}=c$ and $b_{0}=-a$, is given. In this case, $\gamma_{1}$ and $\gamma_{2}$ are given in (4.9), and

$$
1+\gamma_{1}=a, \quad 2+\gamma_{1}+\gamma_{2}=c, \quad \alpha=-1 .
$$

We then confirm that the set of (4.8) and (4.21) agrees with the set of two C-solutions of Kummer's DE given in those books.

\section{Solution of fDE (3.1) for $\sigma=1 / 2$}

In this section, we consider the case of $\sigma=\frac{1}{2}, m=2$, $a_{2} \neq 0, a_{1} \neq 0, a_{0}=0, b_{2}=b_{1}=0$ and $b_{0} \neq 0$.

Then the Equation (3.1) to be solved is

$$
\begin{aligned}
& a_{2} t \cdot{ }_{0} D_{R} u(t)+a_{1} t \cdot{ }_{0} D_{R}^{1 / 2} u(t)+b_{0} u(t)=f(t), \\
& t>0 .
\end{aligned}
$$

Then (3.5) and (3.6) are expressed as

$$
\begin{aligned}
& A(x)=a_{2} x+a_{1} x^{1 / 2}=a_{2} x^{1 / 2}\left(x^{1 / 2}+\alpha\right), \\
& B(x)=b_{0}-a_{2}-\frac{1}{2} a_{1} x^{-1 / 2}, \\
& \hat{v}(x)=0,
\end{aligned}
$$

where $\alpha=a_{1} / a_{2}$.

\subsection{Complementary Solution of (3.7)}

By using (5.2), $B(x) / A(x)$ is expressed as

$$
\frac{B(x)}{A(x)}=x^{-1 / 2}\left(\frac{\gamma_{1}}{x^{1 / 2}}+\frac{\gamma_{2}}{x^{1 / 2}+\alpha}\right),
$$

where 


$$
\gamma_{1}+\gamma_{2}=\frac{b_{0}}{a_{2}}-1, \quad \gamma_{1}=-\frac{1}{2}, \quad \gamma_{2}=\frac{b_{0}}{a_{2}}-\frac{1}{2} .
$$

By (3.8), the C-solution $\hat{\phi}(x)$ of (3.7) is given by

$$
\begin{aligned}
\hat{\phi}(x) & =x^{\gamma_{1}}\left(x^{1 / 2}+\alpha\right)^{2 \gamma_{2}}=x^{\gamma_{1}+\gamma_{2}}\left(1+\alpha x^{-1 / 2}\right)^{2 \gamma_{2}} \\
& =\sum_{n=0}^{\infty}\left(\begin{array}{c}
2 \gamma_{2} \\
n
\end{array}\right) \alpha^{n} x^{-n / 2+\gamma_{1}+\gamma_{2}} .
\end{aligned}
$$

\subsection{Complementary Solution of (3.2) or (5.1)}

The C-solution of (3.2) is given by

$$
\begin{aligned}
u(t) H(t) & =\hat{u}(D) \delta(t)=C_{1} \cdot \hat{\phi}(D) \delta(t) \\
& =C_{1} \sum_{n=0}^{\infty}\left(\begin{array}{c}
2 \gamma_{2} \\
n
\end{array}\right) \alpha^{n} D^{-n / 2+\gamma_{1}+\gamma_{2}} \delta(t) .
\end{aligned}
$$

By Condition B, we have to require

$$
\gamma_{1}+\gamma_{2}=\frac{b_{0}}{a_{2}}-1<0
$$

Then by using (2.9) in (5.7), we obtain

$$
\begin{aligned}
& u(t) H(t)=C_{1} \cdot \phi(t) \\
& =C_{1} \sum_{n=0}^{\infty}\left(\begin{array}{c}
2 \gamma_{2} \\
n
\end{array}\right) \frac{1}{\Gamma\left(n / 2-\gamma_{1}-\gamma_{2}\right)} \alpha^{n} t^{n / 2-\gamma_{1}-\gamma_{2}-1} H(t) .
\end{aligned}
$$

The C-solution of (5.1) is equal to this for $t>0$.

\subsection{Particular Solution of (3.2) or (5.1)}

By using the expressions of $A(x)$ and $\hat{\phi}(x)$ given by (5.2) and (5.6) in (3.9), we obtain the P-solution of (3.7) when the inhomogeneous part is $x^{-\nu}$ :

$$
\begin{aligned}
\hat{u}_{v}^{*}(x) & =-x^{\gamma_{1}}\left(x^{1 / 2}+\alpha\right)^{2 \gamma_{2}} \int^{x} \frac{\xi^{-v}}{a_{2} \xi^{1 / 2+\gamma_{1}}\left(\xi^{1 / 2}+\alpha\right)^{1+2 \gamma_{2}}} \mathrm{~d} \xi+C_{3} \cdot \hat{\phi}(x) \\
& =-\frac{1}{a_{2}} x^{\gamma_{1}+\gamma_{2}}\left(1+\alpha x^{-1 / 2}\right)^{2 \gamma_{2}} \int^{x} \xi^{-1-\gamma_{1}-\gamma_{2}-v}\left(1+\alpha \xi^{-1 / 2}\right)^{-1-2 \gamma_{2}} \mathrm{~d} \xi+C_{3} \cdot \hat{\phi}(x) \\
& =-\frac{1}{a_{2}} \sum_{n=0}^{\infty}\left(\begin{array}{c}
2 \gamma_{2} \\
n
\end{array}\right) \alpha^{n} x^{-n / 2+\gamma_{1}+\gamma_{2}} \int^{x} \sum_{m=0}^{\infty}\left(\begin{array}{c}
-1-2 \gamma_{2} \\
m
\end{array}\right) \alpha^{m} \xi^{-m / 2-\gamma_{1}-\gamma_{2}-1-v} \mathrm{~d} \xi+C_{3} \cdot \hat{\phi}(x) \\
& =\frac{1}{a_{2}} \sum_{n=0}^{\infty}\left(\begin{array}{c}
2 \gamma_{2} \\
n
\end{array}\right) \alpha^{n} x^{-n / 2} \sum_{m=0}^{\infty}\left(\begin{array}{c}
-1-2 \gamma_{2} \\
m
\end{array}\right) \frac{1}{m / 2+\gamma_{1}+\gamma_{2}+v} \alpha^{m} x^{-m / 2-v} \\
& =2 \sum_{n=0}^{\infty}{ }_{n} C_{2 \gamma_{2}, 2 \gamma_{1}+2 v}^{*} \cdot \alpha^{n} x^{-n / 2-v},
\end{aligned}
$$

where ${ }_{n} C_{2 \gamma_{2}, 2 \gamma_{1}+2 v}^{*}$ is defined by (4.11) and is given by (4.12).

By using (4.12) in (5.9), we can show that if the inhomogeneous part is $\frac{1}{\Gamma(v)} t^{\nu-1} H(t)$ for $v>0$, the P-solution of (3.2) is given by

$$
\begin{aligned}
& u_{v}^{*}(t) H(t)=\hat{u}_{v}^{*}(D) \delta(t) \\
& =\frac{1}{a_{2}\left(v+\gamma_{1}+\gamma_{2}\right)} \sum_{n=0}^{\infty} \frac{\left(2 v+2 \gamma_{1}\right)_{n}}{\left(1+2 v+2 \gamma_{1}+2 \gamma_{2}\right)_{n}} \\
& \cdot \frac{1}{\Gamma(n / 2+v)}(-\alpha)^{n} t^{n / 2+v-1} H(t) .
\end{aligned}
$$

This $u_{v}^{*}(t)$ for $t>0$ gives the P-solution of (5.1), when the inhomogeneous part is $\frac{1}{\Gamma(v)} t^{\nu-1}$ for $v>0$.

\section{REFERENCES}

[1] K. Yosida, "The Algebraic Derivative and Laplace's Dif- ferential Equation," Proceedings of the Japan Academy, Vol. 59, Ser. A, 1983, pp. 1-4.

[2] K. Yosida, “Operational Calculus," Springer-Verlag, New York, 1982, Chapter VII.

[3] J. Mikusiński, “Operational Calculus," Pergamon Press, London, 1959.

[4] T. Morita and K. Sato, "Solution of Fractional Differential Equation in Terms of Distribution Theory," Interdisciplinary Information Sciences, Vol. 12, No. 2, 2006, pp. 71-83.

[5] T. Morita and K. Sato, "Neumann-Series Solution of Fractional Differential Equation," Interdisciplinary Information Sciences, Vol. 16, 2010, pp. 127-137.

[6] M. Abramowitz and I. A. Stegun, "Handbook of Mathematical Functions with Formulas, Graphs and Mathematical Tables," Dover Publ. Inc., New York, 1972, Chapter 13.

[7] M. Magnus and F. Oberhettinger, "Formulas and Theorems for the Functions of Mathematical Physics," Chelsea Publ. Co., New York, 1949, Chapter VI. 


\section{Appendix A: Definition of a Distribution in $\mathcal{D}^{\prime}{ }_{R}$ and Its Fractional Integral and}

\section{Derivative}

A right-sided distribution $h(t) \in \mathcal{D}^{\prime}{ }_{R}$ is a functional for which a number $\langle h, g\rangle$ is associated with all $g(t) \in \mathcal{D}_{R}$, where $\mathcal{D}_{R}$ is the space of infinitely differentiable functions which is defined on $\mathbb{R}$ and has a support bounded on the right.

A regular right-sided distribution $h(t) \in \mathcal{D}^{\prime}{ }_{R}$ is a locally integrable function on $\mathbb{R}$, which has a support bounded on the left, and $\langle h, g\rangle$ is given by

$$
\langle h, g\rangle=\int_{-\infty}^{\infty} h(t) g(t) \mathrm{d} t .
$$

Let $g(t) \in \mathcal{D}_{R}$. If $\beta=-v \in \mathbb{R}_{<0}$, the fractional integral $D_{W}^{-v} g(t) \in \mathcal{D}_{R}$ is

$$
D_{W}^{-v} g(t):=\frac{1}{\Gamma(v)} \int_{t}^{\infty}(x-t)^{v-1} g(x) \mathrm{d} x,
$$

and if $\beta \in \mathbb{R}_{>0}$, the fractional derivative $D_{W}^{\beta} g(t) \in \mathcal{D}_{R}$ is given by

$$
D_{W}^{\beta} g(t)=D_{W}^{\beta-n}\left[D_{W}^{n} g(t)\right]
$$

where $n=\lceil\beta\rceil$. We set $D_{W}^{0} g(t)=g(t)$, and

$$
D_{W}^{n} g(t)=(-1)^{n} \frac{\mathrm{d}^{n}}{\mathrm{~d} t^{n}} g(t)
$$

for $n \in \mathbb{Z}_{>0}$.

In this place, we can confirm that the index law

$$
D_{W}^{\beta} D_{W}^{v} g(t)=D_{W}^{\beta+v} g(t)
$$

is valid for every $\beta, v \in \mathbb{R}$.

For a distribution $h(t) \in \mathcal{D}^{\prime}{ }_{R}, \quad D^{\beta} h(t) \in \mathcal{D}^{\prime}{ }_{R}$ for $\beta \in \mathbb{R}$ is defined by

$$
\left\langle D^{\beta} h(t), g(t)\right\rangle=\left\langle h(t), D_{W}^{\beta} g(t)\right\rangle .
$$

The index law (2.8) follows from (A.4) by (A.5).

Dirac's delta function $\delta(t)$ is defined by $D H(t)$, as stated just below Lemma 1, and hence

$$
\begin{aligned}
& \langle\delta(t), g(t)\rangle=\langle D H(t), g(t)\rangle \\
& =\left\langle H(t), D_{W} g(t)\right\rangle=-\int_{0}^{\infty} g^{\prime}(t) \mathrm{d} t=g(0) .
\end{aligned}
$$

It is customary to use the notation:

$$
\langle\delta(t), g(t)\rangle=\int_{-\infty}^{\infty} \delta(t) g(t) \mathrm{d} t=g(0) .
$$

Let $h(t) \in \mathcal{D}_{R}^{\prime} \quad$ and $\quad g(t), f(t) g(t) \in \mathcal{D}_{R}$. Then $f(t) h(t) \in \mathcal{D}_{R}^{\prime}$ is defined by

$$
\langle f(t) h(t), g(t)\rangle=\langle h(t), f(t) g(t)\rangle .
$$

\section{Appendix B: Proof of Lemma 4 for $\beta \in \mathbb{R}_{>0}$}

Here we give a proof of Lemma 4 for $\beta \in \mathbb{R}_{>0}$, with the aid of notations explained in Appendix $\mathbf{A}$.

Let $\beta \in \mathbb{R}_{>0}, \quad n=\lceil\beta\rceil$ and $g(t) \in \mathcal{D}_{R}$. Then

$$
\begin{aligned}
& \left\langle t \cdot D^{\beta} \delta(t), g(t)\right\rangle=\left\langle\delta(t), D_{W}^{\beta}[t \cdot g(t)]\right\rangle \\
& =\left\langle\delta(t), D_{W}^{\beta-n} D_{W}^{n}[t \cdot g(t)]\right\rangle \\
& =\left\langle D^{\beta-n} \delta(t), D_{W}^{n}[t \cdot g(t)]\right\rangle \\
& =\left\langle D^{\beta-n} \delta(t),(-n) \cdot D_{W}^{n-1} g(t)+t \cdot D_{W}^{n} g(t)\right\rangle \\
& =-n\left\langle D^{\beta-1} \delta(t), g(t)\right\rangle+\left\langle t \cdot D^{\beta-n} \delta(t), D_{W}^{n} g(t)\right\rangle .
\end{aligned}
$$

Using Lemma 4 for $\beta-n \in \mathbb{R}_{<0}$ in the last member, we obtain

$$
\begin{aligned}
& \left\langle t \cdot D^{\beta} \delta(t), g(t)\right\rangle \\
& =-n\left\langle D^{\beta-1} \delta(t), g(t)\right\rangle-(\beta-n)\left\langle D^{\beta-n-1} \delta(t), D_{W}^{n} g(t)\right\rangle \\
& =-\beta\left\langle D^{\beta-1} \delta(t), g(t)\right\rangle .
\end{aligned}
$$

Formula (2.12) for $\beta \in \mathbb{R}_{>0}$ follows from this.

\section{Appendix C: Solution of Laplace's DE (3.1) for $\sigma=1$}

We now consider the DE (3.1) for $\sigma=1$ and $m=2$. Then (3.5) and (3.6) are expressed as

$$
\begin{aligned}
& A(x)=a_{2} x^{2}+a_{1} x+a_{0}=a_{2}\left(x+\alpha_{+}\right)\left(x+\alpha_{-}\right), \\
& B(x)=b_{2} x^{2}+\left(b_{1}-2 a_{2}\right) x+b_{0}-a_{1}, \\
& \hat{v}(x)=b_{2} u_{0} x-a_{2} u_{0}+b_{2} u_{1}+b_{1} u_{0},
\end{aligned}
$$

where

$$
\alpha_{ \pm}=\frac{a_{1} \pm \sqrt{a_{1}^{2}-4 a_{2} a_{0}}}{2 a_{2}} .
$$

In solving (3.7), we express $B(x) / A(x)$ as

$$
\frac{B(x)}{A(x)}=\frac{b_{2}}{a_{2}}+\frac{c_{0}+c_{1} x}{A(x)},
$$

where $c_{0}$ and $c_{1}$ are constants. In Section 4.1, we assume that $b_{2}=0$ and obtain the $\mathrm{C}$-solution given by (4.8) which satisfies Condition B. In the presence of the first term on the righthand side of (C.5), we will see that we cannot obtain a solution satisfying Condition B. Hence we have to assume $b_{2}=0$.

\section{Appendix D: Solution of fDE (3.1) for $\sigma=1 / 2$}

In this section, we consider the fDE (3.1) for $\sigma=\frac{1}{2}$ and $m=2$. Then (3.5) and (3.6) are expressed as 
$A(x)=a_{2} x+a_{1} x^{1 / 2}+a_{0}=a_{2}\left(x^{1 / 2}+\alpha_{+}\right)\left(x^{1 / 2}+\alpha_{-}\right),(\mathrm{D} .1)$

$B(x)=b_{2} x+b_{1} x^{1 / 2}+b_{0}-a_{2}-\frac{1}{2} a_{1} x^{-1 / 2}$,

$\hat{v}(x)=b_{2} u_{0}+b_{1} u_{-1 / 2}$,

where $\alpha_{ \pm}$are given by (C.4).

In solving (3.7), we express $B(x) / A(x)$ as

$$
\frac{B(x)}{A(x)}=\frac{b_{2}}{a_{2}}+\left(\frac{b_{1}}{a_{2}}-\frac{b_{2} a_{1}}{a_{2}^{2}}\right) x^{-1 / 2}+\frac{c_{0}+c_{1} x^{-1 / 2}}{A(x)} \text {, (D.4) }
$$

where $c_{0}$ and $c_{1}$ are constants. In Section 5.2, we assume that $b_{2}=b_{1}=0$ and obtain the C-solution given by (5.8) which satisfies Condition B. In the presence of the first two terms on the righthand side of (D.4), we will see that we cannot obtain a solution satisfying Condition B. Hence we have to assume $b_{2}=b_{1}=0$. 